\title{
The Efficiency of Variance Reduction in Manufacturing and Service Systems: The Comparison of the Control Variates and Stratified Sampling
}

\author{
Ergün Eraslan and Berna Dengiz \\ Department of Industrial Engineering, Baskent University, Eskisehir Road 22.km, \\ 06590 Ankara, Turkey \\ Correspondence should be addressed to Ergün Eraslan, eraslan@baskent.edu.tr \\ Received 6 May 2008; Revised 17 November 2008; Accepted 8 February 2009 \\ Recommended by Irina Trendafilova
}

There has been a great interest in the use of variance reduction techniques (VRTs) in simulation output analysis for the purpose of improving accuracy when the performance measurements of complex production and service systems are estimated. Therefore, a simulation output analysis to improve the accuracy and reliability of the output is required. The performance measurements are required to have a narrow and strong confidence interval. For a given confidence level, a smaller confidence interval is supposed to be better than the larger one. The wide of confidence interval, determined by the half length, will depend on the variance. Generally, increased replication of the simulation model appears to have been the easiest way to reduce variance but this increases the simulation costs in complex-structured and large-sized manufacturing and service systems. Thus, VRTs are used in experiments to avoid computational cost of decision-making processes for more precise results. In this study, the effect of Control Variates (CVs) and Stratified Sampling (SS) techniques in reducing variance of the performance measurements of $M / M / 1$ and GI/G/1 queue models is investigated considering four probability distributions utilizing randomly generated parameters for arrival and service processes.

Copyright (C) 2009 E. Eraslan and B. Dengiz. This is an open access article distributed under the Creative Commons Attribution License, which permits unrestricted use, distribution, and reproduction in any medium, provided the original work is properly cited.

\section{Introduction}

Manufacturing systems are processing systems where raw materials are transformed into finished products through a series of workstations. It is important to find an alternative design process to obtain desired performance in a manufacturing system based on management decision. A service system is also a processing system where one or more service facilities are provided to customers, patients, and paperworks. 


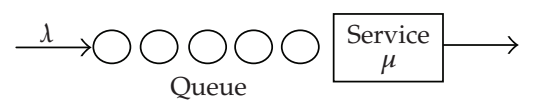

Figure 1: One server one line queue system (M/M/1).

The use of simulation for the modeling of service and manufacturing systems has greatly increased recently in the many areas of application such as health care systems, restaurants, cafeterias, banks, and recreation centers (cinemas, theatres), and many manufacturing systems.

In systems mentioned above, the most widely used queue model is $M / M / 1$. The queue model refers to exponential arrivals and service times with a single server and one line shown in Figure 1. M/M/1 is a good approximation for a large number of queueing systems. There are many systems we encountered in service and production fields where $\mathrm{M} / \mathrm{M} / 1$ model can be used for modeling these systems such as a cashier in a supermarket and a teller in a bank.

$\mathrm{M} / \mathrm{M} / 1$ is Kendall's notation of this queuing model. The first $M$ represents the input process, the second $\mathrm{M}$ the service distribution, and 1 the number of server. The $\mathrm{M}$ implies an exponentially distributed interarrival and service time. The $M / M / 1$ queue system has also unlimited population and First-in First-out (FIFO) queue discipline. On the other hand, if the distributions of arrival and service processes are not Markovian, the one server queue system is named GI/G/1.

Although there are some analytic solutions for these systems, the performance measurements of them represent steady-state behavior. Therefore, in real life applications, the simulation technique is used to compute system performance measures for any time interval. Simulation is more relevant and flexible technique to solve the problems of queue systems in manufacturing and service systems [1].

The queues are used for modeling of manufacturing systems, for example, inventory models, flow line, and JIT production systems. The unbalanced flow line capacity in the manufacturing systems can constitute a product or semiproduct queue which can be usually modeled as $\mathrm{M} / \mathrm{M} / 1$ or GI/G/1. The queues can cause a bottleneck in front of the machines in the job shop (see Figure 2). The delay caused by bottlenecks in manufacturing systems increases the unit cost, decreases the productivity, and which in turn affects the competitiveness of the companies in the market negatively. This is the most common research area of Industrial Engineering in managerial and operational system analyses.

In service systems, the success of a company, besides using the resources efficiently, depends on winning customers and keeping them. Any lost time by customers standing in the queues accounts for loss of profit and usefulness for the service companies.

To address such problems, simulation technique is used as a flexible modeling tool to investigate and solve the queue problems occuring in manufacturing and service systems. Because random samples from probability distributions are used to drive a simulation model, outputs of the simulation model are just particular realization of random variables that may have large variances. For the reasons mentioned, there has been a rapid growth of interest in the use of variance reduction techniques (VRTs) for improving the accuracy of simulation outputs. Thus, the VRTs can be used through the run of the simulation models to obtain more precise results.

Therefore, in this study, we investigated the effect of two different VRTs on the M/M/1 and GI/G/1 queue models. 


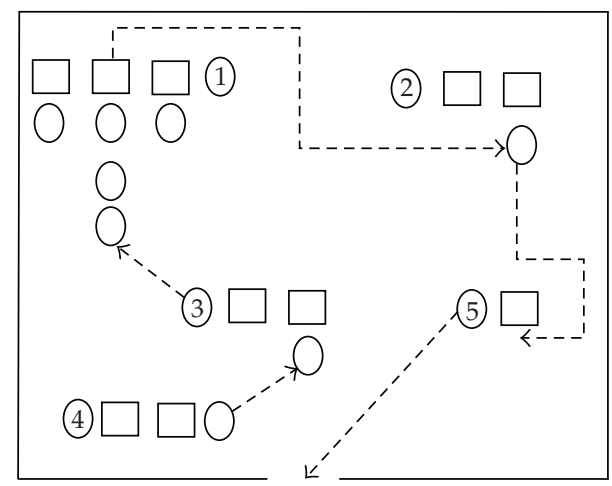

Figure 2: M/M/1 queues of the products/semiproducts in a job shop ( $\square$ represents a server $\bigcirc$ represents a manufacturer of a semiproduct).

The common VRTs are Common Random Variables (CRVs), Antithetic Variables $(\mathrm{AV})$, Control Variates (CV), Stratified Sampling (SS), Importance Sampling (IS), Indirect Estimation (IE), and Conditional Expectation (CE) [2].

The studies on variance reduction (VR) began in the 1950s. In the years before advance computer technology, AV was used in Monte Carlo simulation. Kleijnen was interested in CRV and AV [3]. The CV technique was developed at the end of 1970s and used in a queue simulation by Carson [4], Lavenberg et al. [5], and Wilson and Pristker [6]. Law's formula was the basic of IE and Queue Theory [7]. Cartel and Ignall [8] used this formula in CRV simulation. In the following years, Nelson [9] tried the well-known VRTs in dynamic systems. Until the 1990s, there are several authors that have studied computer simulation of the VRTs, for example, Kleijnen [3], Lavenberg et al. [5], Carson and Law [10], Carter and Ignall [8], Iglehart and Shedler [11], and Wilson and Pristker [6].

In the last decade, VRTs have been used in several areas. Statistics and simulation output analyses are the primary fields and mathematics, chemistry, medicine, biology, quality improvement, portfolio analysis, pricing, flexible manufacturing systems, scheduling, stochastic networks, nuclear chemistry, oceanography, and biophysics, and Markov processes follow them. These studies shortly listed below.

Dengiz et al. [12] used the AV in the stochastic networks, Nava [13] used the VRTs in comparing the simulation models, and Shih and Song [14] in regenerative simulation. Crawford and Gallwey [15] took into account the bias of computerized simulation studies, Dahl [16] in diffusion with CV technique, Vegas et al. [17] in dichotomous response variables, Kawrakow and Fippel [18] in calculating photon dose, Plante [19] in supplier interactions of the companies, Glasserman et al. [20] in estimating of risk values for the investments, Srikant and Whitt [21] in loss model simulation, Taylor and Heragu [22] in workshop flows for shortening operations time in flow shop, Cancela and El Khadiri [23] in increasing the network reliability, Moreni [24] for establishing the pricing options of the companies, Jourdain et al. [25] in polymeric fluids in engineering, and Fumera et al. [26] in bagging optimization. The VRTs have been used in Monte Carlo simulation studies in the recent years by Skowronski and Turner [27] for statistical tolerance synthesis, Pacelli and Ravaioli [28] for semiconductor devices in electronics department, Constantini [29] for reflecting diffusions, Fitzgerald et al. [30] for determining the dynamic levels of systems, and Baker and Hadjiconstantinou [31] for Bolztman equation in fluids mechanic. 
The VR and queuing models being coupled were examined in only a few studies; Görg and Fuß [32] used ATMs in runtimes evaluation, Arsham [33] in calculating the score function estimation, Meles [34] in branching the optimal numbers in mathematical models, Jocabson [35] in harmonic gradient estimator, and Schmeiser and Taaffe [36] in queuing network studies. Sabuncuoglu et al. [37] used two input and two output VRTs to measure the performance of VRTs under finite simulation run lengths and analyzed their effects considering three different types of systems: $\mathrm{M} / \mathrm{M} / 1$, serial production line, and $(\mathrm{S}, \mathrm{S})$ inventory control systems.

Previous research in the area mainly focuses on applications of variance reduction techniques on $\mathrm{M} / \mathrm{M} / 1$ queues. While the first objective of this paper deals with comparison and analysis of the performance of both VRTs (CV, SS) on the simple queue systems such as $M / M / 1$ and GI/G/1, we also investigate the effects of different distributions used for modeling of arrival and service processes of these models utilizing experimental design analysis.

In this study, the average waiting time (AWT) and average number of customers (ANCs) are considered as system performance measurements. CV and SS techniques are used for the variance reduction of simulation outputs. The efficiencies of each technique on the simple queue models are investigated for four different distributions. These distributions which are exponential (in this case, the queue model is called as M/M/1 using Kendall's notation), uniform, triangular, and normal (in these cases the queue model is named as GI/G/1 using Kendall's notation) are used in interarrival times and service times. The randomly selected four parameter sets for four distributions are stated for experiments. The results of factor analysis are given in detail.

This paper will proceed as follows. The next section reviews some VRTs, and experimental analyses are described in Section 3. Finally, the research results and conclusion remarks are summarized in Section 4.

\section{The Variance Reduction Techniques (VRTs)}

The general specifications of CV and SS techniques are reviewed below.

\subsection{Control Variates (CV) Technique}

The basic purpose of $\mathrm{CV}$ is to introduce correlation among observations so as to reduce the variance. Using "Control Variates", true estimation statistics based on a secondary estimation value, and difference between its estimation values are ascertained. With this technique, instead of direct estimation of the parameter, the possible relationship between the problem undertaken and the analytic model is considered (see (2.1)-(2.3)) $[6,38,39]$.

Let $X_{n}$ be a series of the first 100 customer delays in queue, and let $X$ be an output random variable representing the average of the first 100 customer delays in queue:

$$
X=E\left[X_{n}\right]
$$

The value of $X$ is estimated during simulation period.

Let the secondary random variable, $Y$, formed from independent identically distributed random variables (i.e., the service times of the first 99 customers) and its expectation 
$v=E[Y]$ be known because service times are generated from some known input distributions mentioned in Section 1. It is obvious that larger than average service times tend to lead to longer than average delays and vice versa. Thus $Y$ is correlated to $X$, positively [7]. We control the output $X$ using this relation between $X$ and $Y$, where $Y$ is the control variate.

The corrected $X\left(X_{c}\right)$ is obtained from

$$
X_{c}=X-a(Y-v),
$$

where $a>0$ and $Y>v$ if $X_{c}<X, a$ is a constant and takes the same sign with the covariance between $X$ and $Y$ :

$$
\operatorname{Var}\left(X_{c}\right)=\operatorname{Var}[X]+a^{2} \operatorname{Var}[Y]-2 a \operatorname{Cov}[X, Y]
$$

If $2 a \operatorname{Cov}(X, Y)>a^{2} \operatorname{Var}(Y)$ inequality is valid, then $X_{c}$ will have less variability than $X . \operatorname{Cov}(X, Y)$ is estimated through the simulation. $X_{c}$ is calculated using coefficient $a$, then a confidence interval can be built for $X_{c}$ (for detailed information, see Law and Kelton [7]).

\subsection{Stratified Sampling (SS)}

SS technique is such that the heap is divided into stratums. By converting the heaps to stratums which have smaller variances, the problems arising from sensitivity due to big variance are prevented. Here, the determination of the number of stratums is important. Increasing the stratum number results in smaller variance but decreasing the number results in loss of the estimating variance because all data cannot be accounted for in some stratums. Moreover, the more difference between the averages of heaps and stratums the more benefit is supplied. In literature, generally, it is expressed that 3-5 stratums are enough.

There are four kinds of SS available in literature. These are Common Random SS which is used in this study, Proportional SS, Appropriate Sharing Method, and Economical Sharing Method [1].

\section{Experimental Analysis}

We consider a simple queue system which has one waiting line and one server to perform an experimental analysis. Our aim is to determine the effectiveness of CV and SS and how VRTs will avoid computational cost of simulation experiments in obtaining more precise results. The effects of four different probability distributions having randomly generated parameter(s) for arrival and service processes on the precision of simulation output are also examined.

VRTs have two levels as CV and SS. For the arrival and service processes, exponential, uniform, triangular, and normal distributions are selected. Each distribution is assigned to arrival and service processes with randomly selected parameter values as given in Table 3. Comparison and analysis are carried out using statistical output analysis for a single system. Two performance measurements, average waiting time (AWT) and average number of customer (ANC), are considered as system outputs. The reason we use a simple queue system with one line and one server (called $\mathrm{M} / \mathrm{M} / 1$ with exponential distribution for arrival and service process and GI/G/1 with the other general distributions) is to increase the range of application areas in practice and also to obtain mathematical models for them $[2,7,40]$. 
Table 1: A sample application for 5 stratums of SS.

\begin{tabular}{lcccccccc}
\hline \multirow{2}{*}{ Random seeds } & \multirow{2}{*}{ Min } & \multirow{2}{*}{ Max } & \multicolumn{7}{c}{ Customer numbers per stratum } \\
& & & $(0-0.5)$ & $(0.5-1)$ & $(1-1.5)$ & $(1.5-2)$ & $(2+)$ & Total \\
\hline 65000 & 0.0018 & 8.5093 & 28 & 24 & 17 & 11 & 20 & 100 \\
70000 & 0.0008 & 8.2443 & 26 & 26 & 16 & 12 & 20 & 100 \\
75000 & 0.0003 & 6.8270 & 22 & 22 & 18 & 11 & 22 & 100 \\
80000 & 0.0011 & 6.9670 & 23 & 23 & 18 & 11 & 21 & 100 \\
85000 & 0.0052 & 5.4230 & 24 & 24 & 18 & 11 & 19 & 100 \\
90000 & 0.0012 & 6.8600 & 24 & 24 & 16 & 14 & 20 & 100 \\
95000 & 0.0018 & 8.2440 & 24 & 24 & 17 & 11 & 20 & 100 \\
100000 & 0.0045 & 6.9670 & 25 & 25 & 17 & 12 & 19 & 100 \\
105000 & 0.0009 & 5.6330 & 23 & 23 & 19 & 11 & 19 & 100 \\
110000 & 0.0018 & 8.5090 & 23 & 23 & 17 & 13 & 18 & 100 \\
115000 & 0.0012 & 8.3485 & 23 & 23 & 17 & 12 & 21 & 100 \\
120000 & 0.0012 & 8.2443 & 23 & 24 & 18 & 11 & 20 & 100 \\
\hline
\end{tabular}

\subsection{Variance Reduction with $\mathrm{CV}$}

To determine the efficiency of $\mathrm{CV}$ on $\mathrm{M} / \mathrm{M} / 1$ and GI/G/1 models, the simulation code of $\mathrm{M} / \mathrm{M} / 1$ queue model is used [7]. Some necessary modifications are performed and subroutines are used for eight design points. The two levels of VRTs and the four levels of distributions are then tested. The purpose of $\mathrm{CV}$ technique is to combine an appropriate definition of variables, which depend on the service times. These services are run in a controlled environment. During this study, the values of $X_{n}$ (waiting times in queue) are created and reserved in a hidden file, and then the values are used for the remaining steps of $\mathrm{CV}$. The same operations are done for the service times with their mean being $v$. The constant $a$ is estimated from sample depending on the calculated covariance between $X$ and $Y$ using (3.1). Thus, outputs of simulation model, $X$, are adjusted using CV [41]:

$$
a^{*}=\frac{\operatorname{Cov}(X, Y)}{\operatorname{Var}(Y)}
$$

\subsection{Variance Reduction with SS}

The SS technique works under principles of separation of the heap into stratums and reflects the process of VR of each stratum itself on the overall variance. The necessary modifications are performed on the $\mathrm{M} / \mathrm{M} / 1$ simulation code for stratification. The simulation model is run ten times for 5 stratums for this study, considering 100 customers for each, to obtain the sensitivity and small variance. The replications are done via randomly selected 12 seeds shown in Table 1. After the first run of each seed, a repetition is avoided by using the same initials and a second set of random numbers for the second stratum having 100 customers $[1,4]$.

The five stratums are used in this study as well as the balance of number of customers. A sample application of SS is shown in Table 1. The first column shows the random seeds, the second and third columns represent the minimum and maximum values of stratums, and the next five are the frequency of them for the stratification of 100 customers. Here, one heap is 
Table 2: The comparison of the variances of outputs for AWT.

\begin{tabular}{lccc}
\hline VRT techniques & $F_{\text {cal }}$ & $F_{\text {tab }}$ & Results \\
\hline Without CV-with CV & 139 & 2.86 & $H_{o}:$ reject \\
Without SS-with SS & 5.64 & 2.86 & $H_{o}:$ reject \\
With SS-with CV & 24.67 & 2.86 & $H_{o}:$ reject \\
\hline
\end{tabular}

Table 3: The randomly selected parameter sets for four distributions.

\begin{tabular}{lccccc}
\hline Parameter sets & Process & Exponential $(\beta)$ & Uniform $(a, b)$ & Triangular $(a, b, c)$ & Normal $\left(\mu, \sigma^{2}\right)$ \\
\hline \multirow{2}{*}{ Set 1} & Arrival & 1 & 1,2 & $1,2,3$ & $0.5,1$ \\
& Service & 0.5 & 1,3 & $2,3,4$ & $1,1.5$ \\
\hline \multirow{2}{*}{ Set 2} & Arrival & 1.5 & & $0.5,1.5$ \\
& Service & 1 & 2,4 & 1,2 \\
\hline \multirow{2}{*}{ Set 3 } & Arrival & 2 & & 1,4 & 1,2 \\
& Service & 1.5 & 2,3 & $1,2,4$ & $0.5,2$ \\
\hline \multirow{2}{*}{ Set 4} & Arrival & 2.5 & & & $1.5,2$ \\
& Service & 2 & & & $1,2.5$ \\
\hline
\end{tabular}

converted to five stratums which have small variances. Thus, sensitivity problems based on big variances are prevented. As seen in the table, the application of this technique is difficult, as it takes a very long run time.

\subsection{Computational Results of VRTs}

To ascertain the effects of the techniques on the considered queuing models, $F$ hypothesis tests are used on the variance data obtained by applying the CV and SS techniques. The hypotheses are constructed as follows:

Hypothesis

$$
\begin{aligned}
& H_{o}: \sigma_{1}^{2}=\sigma_{2}^{2}, \\
& H_{1}: \sigma_{1}^{2}<\sigma_{2}^{2}, \\
& F_{\text {cal }}=Y>F_{\text {tab }}=X,
\end{aligned}
$$

where $\sigma_{1}$ and $\sigma_{2}$ are the variances of the outputs obtained with and without VRTs, respectively. In the confidence level of $95 \%(\alpha=0.05)$, the calculated $F$ value $\left(F_{\text {cal }}\right)$ is greater than $F$ table value $\left(F_{\text {tab }}\right)$ which means the $H_{o}$ hypothesis will be rejected. Thus, the variance of the output of the simulation model (the performance measurement of the system) obtained with VRT is smaller. The experiments are performed with a randomly selected parameter set ( 2 for arrival and 1.5 for service processes). The exponential distribution is considered for $\mathrm{F}$ tests where $m=n=12$.

As shown in Table 2 both of the techniques reduce variance statistically, and the CV technique is more efficient than $\mathrm{SS}$ for the $\mathrm{M} / \mathrm{M} / 1$ queue with a randomly selected parameter set. Similar results are also obtained for GI/G/1 queue system using the uniform, triangular, and normal distributions. 
Table 4: The variances of AWT.

\begin{tabular}{lcccc}
\hline Levels & 1.Exponential & 2.Uniform & 3.Triangular & 4.Normal \\
\hline \multirow{3}{*}{ 1.Control variates } & 0.000010 & 0.0715000 & 0.0000398 & 0.0000155 \\
& 0.000780 & 0.0063840 & 0.0010400 & 0.0001010 \\
& 0.039400 & 0.0000188 & 0.0124800 & 0.0000254 \\
& 1.503000 & 0.0824800 & 0.0007170 & 0.0000939 \\
\hline \multirow{3}{*}{ 2.Stratified sampling } & 0.105 & 0.395 & 0.432 & 1.541 \\
& 1.012 & 0.492 & 0.386 & 1.224 \\
& 0.972 & 1.415 & 0.664 & 1.238 \\
& 2.430 & 0.162 & 0.531 & 1.127 \\
\hline
\end{tabular}

Table 5: The variances of ANC.

\begin{tabular}{lcccc}
\hline Levels & 1.Exponential & 2.Uniform & 3.Triangular & 4.Normal \\
\hline \multirow{3}{*}{ 1.Control variates } & 0.0000001 & 0.0003459 & 0.0000001 & 0.0000002 \\
& 0.0000223 & 0.0000663 & 0.0000007 & 0.0000032 \\
& 0.0129500 & 0.0000000 & 0.0000191 & 0.0000003 \\
& 0.0828200 & 0.0001932 & 0.0000023 & 0.0000010 \\
\hline \multirow{3}{*}{ 2.Stratified sampling } & 0.536 & 0.167 & 0.113 & 5.869 \\
& 1.592 & 0.537 & 0.045 & 2.664 \\
& 0.669 & 0.167 & 0.088 & 2.177 \\
& 0.223 & 0.026 & 0.107 & 1.924 \\
\hline
\end{tabular}

\subsection{The Effects of VRTs and Distributions on the Output Variance}

As stated in the previous sections, two factors are considered, and the effects of these factors are investigated on the system performance measurements.

Factor settings are as follows.

(1) VRTs: this factor is tested in experimental design in two levels being CV and SS.

(2) The distribution type of arrival and service processes: this factor is tested in four levels: exponential (for this case queue system is called $\mathrm{M} / \mathrm{M} / 1$ ), uniform, triangular, and normal (for these three distributions, queue systems are called GI/G/1).

The considered system performance measurements are AWT and ANC.

Since this study contains two factors with two and four levels, respectively, $2 \times$ $4=8$ design points are required in case of full factorial design. Four replications are made for each design point, so 32 experiments are performed. The results of the experimentation are analyzed by ANOVA. The validation of ANOVA results depends on normality and independence for the error components. This is performed by MINITAB by observing the standardized residual plot graphs. The assumptions are obtained using relevant transformations to the variance data.

These operations are performed for each considered performance measurements of the queue model. The four variance values obtained from different parameter sets for AWT and ANC are stated for four distributions used with CV and SS in Tables 4 and 5, respectively. 
The ANOVA results given in Table 6 for AWT and Table 7 for ANC provide the followings.

(i) The main factor VRTs are statistically significant for AWT, others are not.

(ii) The main factor VRTs, the type of distributions, and their interactions are statistically significant for ANC.

(iii) Conversely, the effect of $\mathrm{CV}$ technique on the performance measurements is stronger than the effect of SS technique. The CV technique results in smaller variance for both considered performance measurements; the difference in VR can be easily seen $(P<.05)$.

(iv) The investigation of interaction between distribution types and the VRTs show that interaction is efficient in VR technique, only for the ANC. The smallest mean belongs to the first level of the first factor, that is, CV, and the third level of the second factor, that is, triangular distribution, shown in Tables 4 and 5.

\section{Discussion and Conclusions}

Queue systems are widely used in various fields in manufacturing and the service industry. The system analysis of the queues for both industries is one of the most highly research problems in Industrial Engineering. These analyses are mainly performed by simulation technique. Simulation output analysis is used to improve the accuracy and the reliability of the performance measures of systems. For a given confidence level, a smaller confidence interval is supposed to be better than the larger one. The wide of the confidence interval will depend on variance. Generally, increased replication of the simulation model seems to be the easiest way to reduce variance but this increases the simulation costs. Therefore, VRTs are used in experiments to avoid computational cost.

In this study, the effects of CV and SS techniques were investigated for queues (with one waiting line and one service) occurring in manufacturing and service. The effects of the two factors are investigated using the experimental design analysis in reducing variance. The first factor, VRT, with two levels (CV and SS) and the second factor, distributions, with four levels (exponential, uniform, triangular, and normal) are considered with ANOVA.

The ANOVA results show that the main factor VRTs, the type of distributions, and their interactions are statistically significant for ANC. Conversely, VRTs are statistically significant for AWT; the other factors are not.

The effect of the CV technique on the performance measurements is stronger than the effect of the SS technique. The CV technique results in smaller variance for both considered performance measurements; the difference in VR can be easily seen $(P<.05)$. It can be concluded that distribution types and VRTs jointly affect variance of the ANC measure but do not affect AWT.

The smallest mean belongs to the first level of the first factor (i.e., CV) and the third level to the second factor (i.e., triangular distribution), a combination resulting in higher efficiency.

The results underline that both CV and SS VRTs reduce variance quite efficiently in the $95 \%$ confidence level. $80 \%$ of the overall variance reduction is obtained using CV technique and $43 \%$ of using SS technique.

The further results based on the design of experiment demonstrate that if the considered system is $\mathrm{M} / \mathrm{M} / \mathrm{1}, \mathrm{CV}$ technique is efficient. If the considered model of a system 
Table 6: The ANOVA output for AWT.

\begin{tabular}{|c|c|c|c|c|c|c|}
\hline \multicolumn{7}{|c|}{ General linear model: Var versus Tech; Dist } \\
\hline Factor & Type levels & Values & & & & \\
\hline Tech & Fixed & $2 \mathrm{cv}$ ss & & & & \\
\hline Dist & Fixed & 4 ex un tr nc & & & & \\
\hline \multicolumn{7}{|c|}{ Analysis of variance for Var, using adjusted SS for tests } \\
\hline Source & DF & Seq SS & Adj SS & Adj MS & $F$ & $P$ \\
\hline Tech & 1 & 4.29330 & 4.29330 & 4.29330 & 48.73 & .000 \\
\hline Dist & 3 & 0.42013 & 0.42013 & 0.14004 & 1.59 & .218 \\
\hline Tech $*$ Dist & 3 & 0.42165 & 0.42165 & 0.14055 & 1.60 & .217 \\
\hline Error & 24 & 2.11432 & 2.11432 & 0.08810 & & \\
\hline Total & 31 & 7.24940 & & & & \\
\hline
\end{tabular}

Table 7: The ANOVA output for ANC.

\begin{tabular}{lcccccc}
\hline \multicolumn{7}{c}{ General linear model: Var versus Tech; Dist } \\
\hline $\begin{array}{l}\text { Factor } \\
\text { Tech }\end{array}$ & $\begin{array}{c}\text { Type levels } \\
\text { Fixed }\end{array}$ & \multicolumn{1}{c}{ Values } \\
Dist & Fixed & \multicolumn{2}{c}{ cr ss } \\
\hline \multicolumn{7}{c}{ Analysis of variance for Var, using adjusted SS for tests } \\
\hline Source & DF & Seq SS & Adj SS & Adj MS & F & $P$ \\
Tech & 1 & 4.9823 & 4.9823 & 4.9823 & 97.65 & .000 \\
Dist & 3 & 2.5163 & 2.5163 & 0.8388 & 16.44 & .000 \\
Tech*Dist & 3 & 2.5459 & 2.5459 & 0.8486 & 16.63 & .000 \\
Error & 24 & 1.2245 & 1.2245 & 0.0510 & & \\
Total & 31 & 11.2689 & & & & \\
\hline
\end{tabular}

is GI/G/1 and its source of randomness (arrival and service distributions) is fitted using triangular distribution, then the $\mathrm{CV}$ technique is preferable to obtain more beneficial results with smaller variance. The results are only valid under the current experiments for the selected two VRTs and four distributions.

It is supposed that it is more useful to extend this research considering other VRTs to investigate and solve the problems of queuing systems in the manufacturing and service systems area as future research.

\section{References}

[1] N. Iscil, Sampling Methods, Die Library, Ankara University, Ankara, Turkey, 1977.

[2] B. L. Nelson, "Decomposition some well-known variance reduction techniques," Journal of Statistics and Computer Simulation, vol. 23, no. 3, pp. 183-209, 1986.

[3] J. P. C. Kleijnen, "Antithetic variates, common random numbers and computation time allocation in simulation," Management Science, vol. 21, no. 10, pp. 1176-1185, 1975.

[4] J. S. Carson, "Variance reduction techniques for simulated queuing process," Tech. Rep. 78-8, Department of Industrial and Systems Engineering, University of Wisconsin, Madison, Wis, USA, 1978.

[5] S. S. Lavenberg, T. L. Moeller, and P. D. Welch, "Statistical results on control variates with queuing network simulation," Operational Research, vol. 30, no. 1, pp. 182-202, 1982. 
[6] J. R. Wilson and A. A. B. Pristker, "Variance reduction in queuing simulation using generalized concomitant variables," Journal of Statistical Computation and Simulation, vol. 19, no. 2, pp. 129-153, 1984.

[7] A. M. Law and W. D. Kelton, Simulation Modeling and Analysis, McGraw-Hill Series in Industrial Engineering and Management Science, McGraw-Hill, New York, NY, USA, 2nd edition, 1982.

[8] G. Carter and E. J. Ignall, "Virtual measure: a variance reduction technique for simulation," Management Science, vol. 21, no. 6, pp. 607-616, 1975.

[9] B. L. Nelson, "A perspective on variance reduction in dynamic simulation experiments," Communications in Statistics. Simulation and Computation, vol. 16, no. 2, pp. 385-426, 1987.

[10] J. S. Carson and A. M. Law, “Conservation equations and variance reduction in queueing simulations," Operations Research, vol. 28, no. 3, part I, pp. 535-546, 1980.

[11] D. L. Iglehart and G. S. Shedler, "Simulation output analysis for local area computer networks," Acta Informatica, vol. 21, no. 4, pp. 321-338, 1984.

[12] B. Dengiz, A. S. Selcuk, and F. Altiparmak, "Antithetic variate in simulation of stochastic networks: experimental evaluation," in Proceedings of International AMSE Conference on Signals, Data Systems, vol. 2, pp. 41-49, AMSE Press, Calcutta, India, December 1992.

[13] M. P. Nava, "On the use of variance reduction techniques when comparing simulation systems at the steady state," Computers E Industrial Engineering, vol. 29, no. 1-4, pp. 483-487, 1995.

[14] N.-H. Shih and W. T. Song, "Correlation-inducing variance reduction in regenerative simulation," Operations Research Letters, vol. 19, no. 1, pp. 17-23, 1996.

[15] J. W. Crawford and T. J. Gallwey, "Bias and variance reduction in computer simulation studies," European Journal of Operational Research, vol. 124, no. 3, pp. 571-590, 2000.

[16] F. A. Dahl, "Variance reduction for simulated diffusions using control variates extracted from state space evaluations," Applied Numerical Mathematics, vol. 43, no. 4, pp. 375-381, 2002.

[17] E. Vegas, J. del Castillo, and J. Ocaña, "Efficiency and exponential models in a variance-reduction technique for dichotomous response variables," Journal of Statistical Planning and Inference, vol. 85, no. 1-2, pp. 61-74, 2000.

[18] I. Kawrakow and M. Fippel, “Investigation of variance reduction techniques for Monte Carlo photon dose calculation using XVMC," Physics in Medicine and Biology, vol. 45, no. 8, pp. 2163-2183, 2000.

[19] R. Plante, "Allocation of variance reduction targets under the influence of supplier interaction," International Journal of Production Research, vol. 38, no. 12, pp. 2815-2827, 2000.

[20] P. Glasserman, P. Heidelberger, and P. Shahabuddin, "Variance reduction techniques for estimating value-at-risk," Management Science, vol. 46, no. 10, pp. 1349-1364, 2000.

[21] R. Srikant and W. Whitt, "Variance reduction in simulations of loss models," Operations Research, vol. 47, no. 4, pp. 509-523, 1999.

[22] G. D. Taylor and S. S. Heragu, "A comparison of mean reduction versus variance reduction in processing times in flow shops," International Journal of Production Research, vol. 37, no. 9, pp. 1919$1934,1999$.

[23] H. Cancela and M. El Khadiri, "The recursive variance-reduction simulation algorithm for network reliability evaluation," IEEE Transactions on Reliability, vol. 52, no. 2, pp. 207-212, 2003.

[24] N. Moreni, "A variance reduction technique for American option pricing," Physica A, vol. 338, no. 1-2, pp. 292-295, 2004.

[25] B. Jourdain, C. Le Bris, and T. Lelièvre, "On a variance reduction technique for micro-macro simulations of polymeric fluids," Journal of Non-Newtonian Fluid Mechanics, vol. 122, no. 1-3, pp. 91106, 2004.

[26] G. Fumera, F. Roli, and A. Serrau, "Dynamics of variance reduction in bagging and other techniques based on randomisation," in Proceedings of the 6th International Workshop on Multiple Classifier Systems (MCS '05), vol. 3541 of Lecture Notes in Computer Science, pp. 316-325, Seaside, Calif, USA, June 2005.

[27] V. J. Skowronski and J. U. Turner, "Using Monte-Carlo variance reduction in statistical tolerance synthesis," Computer-Aided Design, vol. 29, no. 1, pp. 63-69, 1997.

[28] A. Pacelli and U. Ravaioli, "Analysis of variance-reduction schemes for ensemble Monte Carlo simulation of semiconductor devices," Solid-State Electronics, vol. 41, no. 4, pp. 599-605, 1997.

[29] C. Costantini, "Variance reduction by antithetic random numbers of Monte Carlo methods for unrestricted and reflecting diffusions," Mathematics and Computers in Simulation, vol. 51, no. 1-2, pp. $1-17,1999$.

[30] M. Fitzgerald, P. P. Picard, and R. N. Silver, "Monte Carlo transition dynamics and variance reduction," Journal of Statistical Physics, vol. 98, no. 1-2, pp. 321-345, 2000. 
[31] L. L. Baker and N. G. Hadjiconstantinou, "Variance reduction for Monte Carlo solutions of the Boltzmann equation," Physics of Fluids, vol. 17, no. 5, Article ID 051703, 4 pages, 2005.

[32] C. Görg and O. Fuß, "Comparison and optimization of restart run time strategies," AEÜ-International Journal of Electronics and Communications, vol. 52, no. 3, pp. 197-204, 1998.

[33] H. Arsham, "Stochastic optimization of discrete event systems simulation," Microelectronics Reliability, vol. 36, no. 10, pp. 1357-1368, 1996.

[34] V. B. Meles, "Branching techniques for Markov-chain simulation (finite-state case)," Statistics, vol. 25, no. 2, pp. 159-171, 1994.

[35] S. H. Jocabson, "Variance and bias reduction techniques for harmonic gradient estimator," Applied Mathematics and Computation, vol. 55, no. 2-3, pp. 153-186, 1993.

[36] B. W. Schmeiser and M. R. Taaffe, "Time-dependent queueing network approximations as simulation external control variates," Operations Research Letters, vol. 16, no. 1, pp. 1-9, 1994.

[37] I. Sabuncuoglu, M. M. Fadiloglu, and S. Celik, "Variance reduction techniques: experimental comparison and analysis for single systems," IIE Transactions, vol. 40, no. 5, pp. 538-551, 2008.

[38] R. Añonuevo and B. L. Nelson, "Automated estimation and variance reduction via control variates for infinite-horizon simulations," Computers \& Operations Research, vol. 15, no. 5, pp. 447-456, 1988.

[39] R. Y. Rubinstein and R. Marcus, "Efficiency of multivariate control variates in Monte Carlo simulations," Operations Research, vol. 33, no. 3, pp. 661-677, 1985.

[40] A. Alan and B. Pristker, Simulation and Slam II, John Wiley \& Sons, New York, NY, USA, 1986.

[41] A. A. Mohammed, D. Gross, and D. R. Miller, "Control variates models for estimating transient performance measures in repairable items systems," Management Science, vol. 38, no. 3, pp. 388-399, 1992. 


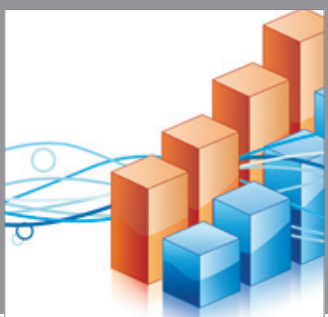

Advances in

Operations Research

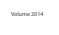

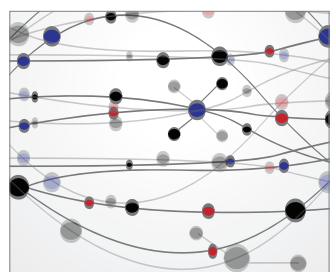

\section{The Scientific} World Journal
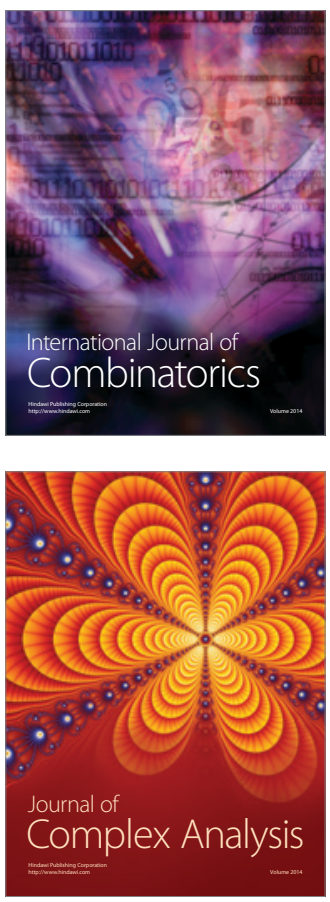

International Journal of

Mathematics and

Mathematical

Sciences
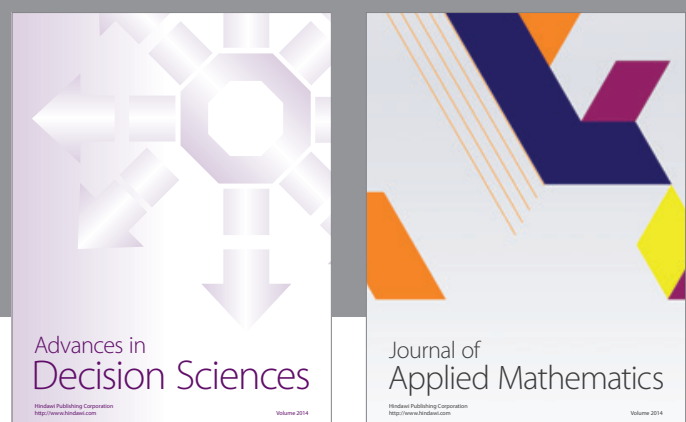

Journal of

Applied Mathematics
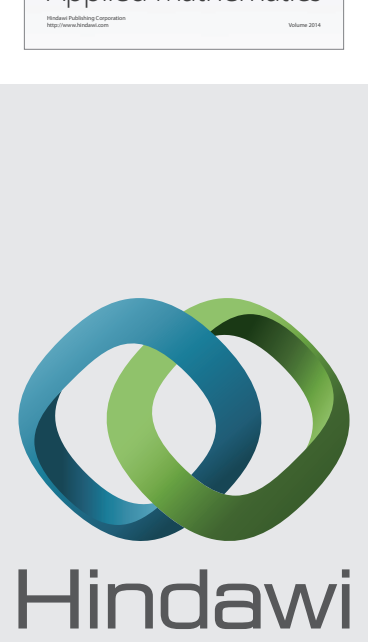

Submit your manuscripts at http://www.hindawi.com
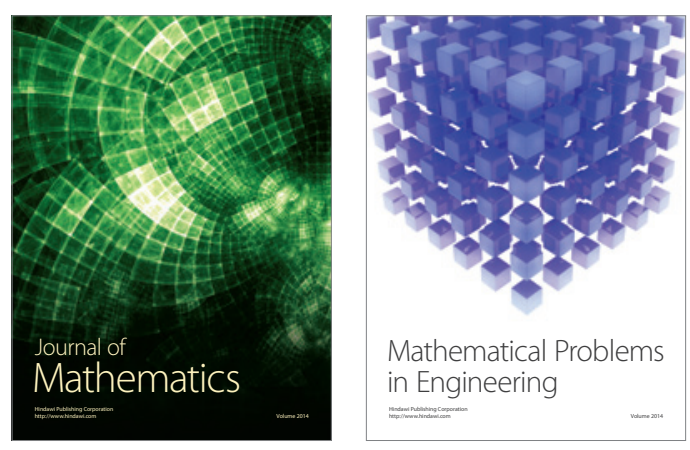

Mathematical Problems in Engineering
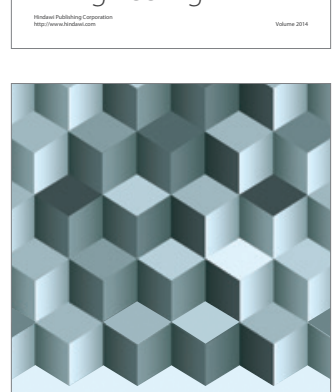

Journal of

Function Spaces
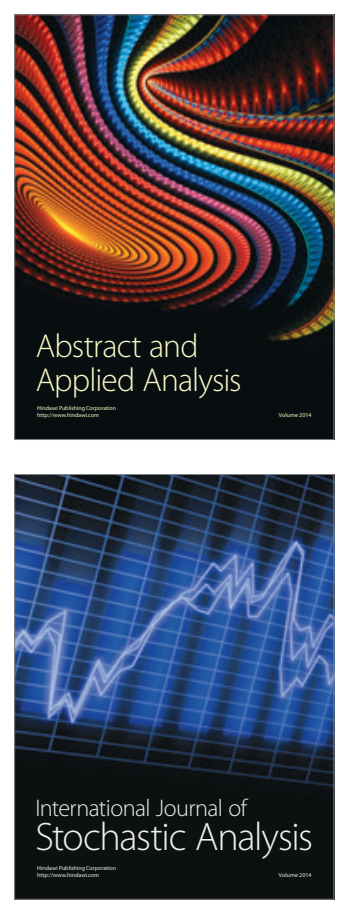

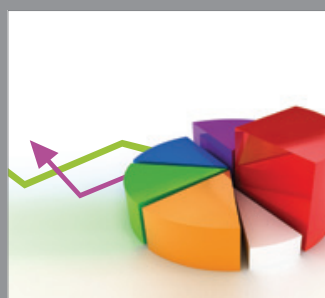

ournal of

Probability and Statistics

Promensencen
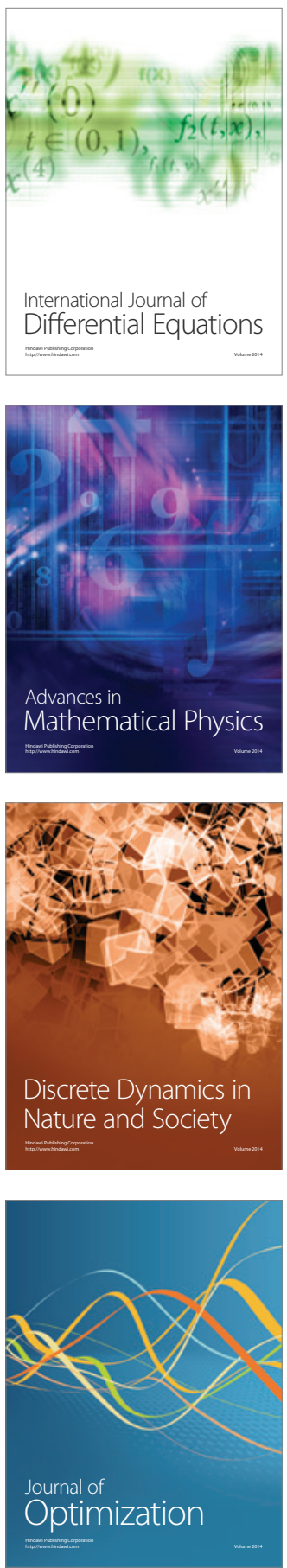